\title{
Os líquens como bioindicadores de poluição atmosférica no município de Uberaba, Minas Gerais, Brasil
}

The lichens as bioindicators of air pollution in county of Uberaba, Minas Gerais, Brazil.

\author{
William Raimundo Costa'; Marina Farcic Mineo² \\ 1,2 Associação de Preservação e Pesquisa Ambiental Vale Encantado, APPA - VE.
}

\section{Resumo}

Os líquens são reconhecidos como ótimos bioindicadores de qualidade do ar, em função de sua alta sensibilidade à poluição atmosférica. $O$ presente trabalho apresenta os dados obtidos no monitoramento passivo da qualidade do ar em sete diferentes pontos da cidade de Uberaba, MG. Foram identificados 42 táxons, distribuídos em 14 famílias e 23 gêneros, sendo que para as áreas onde existe maior incidência de atividades potencialmente poluidoras observou-se as menores diversidades de espécies. Ao final do estudo, concluiu-se que houve discrepância de diversidade liquênica entre as unidades amostrais estudadas. Concluiu-se ainda que o presente trabalho tem potencial para contribuir com o aprimoramento das políticas públicas de controle da poluição atmosférica, bem como para um zoneamento mais eficiente do município.

Palavras-chaves: biomonitoramento; liquenologia; qualidade do ar.

\begin{abstract}
Lichens are recognized as excellent bioindicators of air quality, due to its high sensitivity to air pollution. This study presents data obtained in the passive monitoring of air quality in seven different places in the city of Uberaba, MG. Were identified 42 taxa, distributed in 14 families and 23 genera. Was observed for the areas where there is an increased incidence of potentially polluting activities, the smaller species diversity. At the end of this study concludes that was great diversity Lichen discrepancy between units sample studied. It was also concluded that the presents findings may contribute to the improvement of public policies to control air pollution, as well as a more efficient municipal zoning.
\end{abstract}

Keywords: biomonitoring; lichenology; air quality. 


\section{INTRODUÇÃO}

A poluição atmosférica constitui-se em um dos principais problemas dos grandes centros urbanos. Desde a revolução industrial no século XVIII a atmosfera vem recebendo enorme carga de poluentes. A referida poluição antes era gerada com a queima de carvão. Atualmente decorre dos gases emanados das indústrias, dentre outros, bem como de escapamentos de milhões de veículos em torno do mundo, dos quais segundo o American Petrol Institute (API, 2011), mais de 90\% utilizam combustíveis derivados do petróleo.

O monitoramento da poluição atmosférica a curto, médio e longo prazo pode ser feito sem a necessidade de utilizar equipamentos sofisticados e de alto custo, com auxílio dos bioindicadores (ELIASARO, et al, 2009). É possível se avaliar os níveis de poluição atmosférica analisando, dentre diversos bioindicadores, a diversidade de líquens presentes em determinada área. Os líquens, como não dispõem de meios de excreção, mostram-se particularmente suscetíveis e sensíveis a compostos tóxicos, sendo mesmo o crescimento de seu talo um dos mais sensíveis indicadores do ar poluído (HAVEN, 2010).

Anatomicamente, os líquens não possuem estomas, nem cutícula, o que significa que gases e componentes do ar podem ser absorvidos e alcançar rapidamente as células do componente fotobionte, sendo que a ausência dessas estruturas determina também sua incapacidade para excretar substâncias tóxicas absorvidas, ou ainda a possibilidade de selecionar sua absorção (MARTINS, 2008; VALÊNCIA \&CEBALLOS,2002; MARTINS et al, 2008). A natureza dual da associação liquênica e sua sensibilidade a distúrbios ambientais, faz com que os liquens tenham um grande potencial como bioindicadores. Se o delicado balanço entre os simbiontes é quebrado, isto pode levar a morte do talo (ELIASARO, 2010).

No município de Uberaba, Minas Gerais, não existem estudos ou levantamentos sobre a diversidade liquênica ou acerca do impacto da poluição atmosférica sobre essa biodiversidade.

Desta forma, o presente trabalho tem como proposta a utilização de metodologia simples e barata de monitoramento preliminar da qualidade do ar, com base em estudo da diversidade de líquens, utilizando-os como bioindicadores de poluição atmosférica. Além disso, o estudo irá contribuir para o conhecimento da diversidade da flora liquênica da cidade de Uberaba-MG.

\section{METODOLOGIA}

\section{Áreas de estudo}

O presente estudo foi conduzido no município de Uberaba, Minas Gerais. A cidade possui população de 295.988 habitantes, distribuída em uma área de $4.524 \mathrm{~km} 2$, e apresenta uma frota de 148.599 veículos, segundo dados do IBGE (2012).

Para a realização do levantamento das espécies de liquens do município, foram estabelecidos sete pontos de coletas. Os pontos de coleta foram distribuídos desde a Reserva Particular do Patrimônio Natural Vale Encantado (RPPNVE), até o Distrito Industrial III (DI-III), da cidade de Uberaba - MG, distantes aproximadamente 50 quilômetros, passando por áreas com maior e menor grau de emissão de poluentes atmosféricos. A referida distribuição foi elaborada de forma a se avaliar a diversidade liquênica em área preservada (RPPNVE), passando por pontos no centro comercial da cidade, local de maior fluxo de veículos motorizados, terminando em área onde os níveis de poluição atmosférica possivelmente são superiores aos demais pontos (DI-III). A distribuição dos pontos foi elaborada de forma a se avaliar a diversidade liquênica ao longo de um gradiente de poluição atmosférica.

Situada a 30 quilômetros ao Norte do centro da cidade de Uberaba-MG (FIGURAS 1 e 2), a RPPNVE, 19³3'10.94" S e 47 $54^{\circ} 00.04$ " $\mathrm{O}$, primeiro ponto de coleta do presente trabalho, foi instituída pela portaria IEF 070/2004, publicada em 14 de maio do ano de 2004 no Diário Oficial do Estado de Minas Gerais. Conta com aproximadamente quarenta hectares possuindo fitofisionomias típicas de Cerrado (lato sensu). O clima da região do tipo Aw, segundo classificação de Koppen, caracterizado por apresentar duas estações bem definidas sendo que a estação seca ocorre de abril a setembro, ao passo que a estação chuvosa ocorre de outubro a março (RAIMUNDO COSTA \& FARIA, 2011). A precipitação pluviométrica média para a região é de 1584,2 mm, segundo dados da Estação Experimental Getúlio Vargas, da Empresa de Pesquisa Agropecuária de Minas Gerais (EPAMIG), localizada no município de Uberaba, Estado de Minas Gerais, em convênio com o Instituto Nacional de Meteorologia (INMET). As coletas realizadas no ponto de coleta 1 se deram em área de fitofisionomia típica de Cerrado, denominada cerrado stricto senso, caracterizada por possuir árvores retorcidas, inclinadas e de pequeno porte (RAIMUNDO COSTA \& FARIA, op.cit). 
O segundo ponto de coleta $\left(19^{\circ} 43^{\prime} 58^{\prime \prime} \mathrm{S}\right.$ $47^{\circ} 54^{\prime} 43^{\prime \prime}$ ) se caracteriza por ser área tipicamente urbana, residencial, com comércio reduzido (FIGURAS 3 e 4). Possui em suas proximidades um reservatório de água tratada do Centro Operacional de Desenvolvimento e Saneamento de Uberaba (CODAU), e apresenta moderado fluxo de veículos, uma vez que esse ponto da cidade é rota de acesso à $M G$ - 190, sentido Uberaba Nova Ponte.

O terceiro ponto de coleta, a Praça da

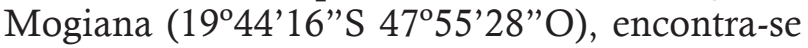
área urbana residencial com vasto comércio alimentício, apresentando inúmeros bares e lanchonetes (FIGURAS 5 e 6). No local existe a Estação Ferroviária de Uberaba, hoje administrada pela empresa Ferrovia Centro Atlântica (FCA), a qual trabalha apenas com transporte de cargas. Nesse mesmo local estão sendo realizadas as obras para a edificação da Sede do Arquivo Público de Uberaba. A área apresenta fluxo reduzido de veículos durante o dia, ao passo que no período noturno apresenta fluxo mais acentuado em virtude do comércio no local.

O quarto ponto de coleta $\left(19^{\circ} 44^{\prime} 52^{\prime \prime} \mathrm{S}\right.$ $\left.47^{\circ} 56^{\prime} 08^{\prime \prime} \mathrm{O}\right)$ apresenta área urbana com intensa atividade comercial (área central da cidade). Localizada no centro da cidade, a Praça Henrique Krugger apresenta intenso fluxo de veículos, possuindo congestionamentos em horários críticos, devido ao alto número de veículos particulares somados ao tráfego de veículos de transporte coletivo (FIGURAS 7 e 8). O local abriga a sede da Empresa Correios e Telégrafos (ECT) no município.

$\mathrm{O}$ quinto ponto de coleta se encontra no Parque Municipal Mata do Ipê (19 $45^{\prime} 43^{\prime \prime}$ S 4756'98"O). Parque de arborização densa (FIGURAS 9 e 10), com árvores de madeira de lei, como aroeira, pau-ferro, paineiras, jatobá, bálsamo, entre outras. É entrecortado por trilhas e possui uma área de lazer de aproximadamente $35.000 \mathrm{~m}^{2}$, segundo informações obtidas no site da Prefeitura Municipal de Uberaba (PMU, 2012), e está inserido em área urbana residencial com comércio moderado, apresentando fluxo de veículos moderado, tendo em vista a proximidade da Secretaria Municipal de Saúde da Cidade de Uberaba.

O sexto ponto de coletas é o Parque de Exposições Fernando Costa (1946'09"S 4756'43”O), de propriedade da Associação Brasileira dos Criadores de Zebu (ABCZ). A área se mostra amplamente arborizada (FIGURAS 11 e 12), com pouco comércio fixado no local, sendo que nos meses de maio e setembro ocorrem, respectivamente, as feiras pecuárias conhecidas como Expozebu e Expoinel.
Nesses meses o comércio na região, bem como a movimentação de pessoas e o fluxo de veículos é bastante intenso. O Parque de Exposições Fernando Costa, embora seja amplamente arborizado, com seus jardins bem cuidados, está inserido em área com grande fluxo de veículos, pois, além de apresentar em suas proximidades o Campus II do Instituto Federal do Triângulo Mineiro (IFTM), é uma das vias de acesso para o Campus II da universidade de Uberaba (UNIUBE), rota para a Faculdade Talentos Humanos (FACTHUS), e ainda é rota para boa parte dos veículos que trafegam em direção à BR - 050, sentido Uberlândia - Uberaba. O sétimo ponto de coleta, o DI-III (1958'54"S 4753'09'O), caracteriza-se por ser formado por indústrias químicas, de fertilizantes, transportadoras e distribuidoras de combustíveis (FIGURAS 13 e 14). Possui área de 18.430.570,00 $\mathrm{m}^{2}$, localizando-se às margens do Rio Grande na divisa com os Estados de MG e SP, a $20 \mathrm{~km}$ do centro da cidade, sendo seu acesso pela Avenida Municipal Filomena Cartafina. Possui diversas indústrias químicas que fabricam insumos agrícolas dentre diversos outros produtos. Referidas indústrias totalizam $13(86,7 \%)$, de um total de 15 instaladas no local, considerando-se aquelas de grande porte. As demais se resumem em transportadoras de derivados de petróleo e beneficiamento de cana-de-açúcar.

\section{coletas de dados}

Partindo da premissa de que comparações dos índices de diversidade liquênica de áreas preservadas com áreas potencialmente poluídas, podem estabelecer variações que determinem o grau de modificações acarretadas em virtude da poluição atmosférica, iniciou-se o desenvolvimento do presente estudo promovendo o levantamento da diversidade de líquens de uma parcela de 10×10m em área da Reserva Particular do Patrimônio Natural Vale Encantado (RPPNVE). Para tanto, foram considerados forófitos com DAP (Diâmetro a Altura do Peito), acima de $10 \mathrm{~cm}$.

Durante os levantamentos da flora liquênica em parcela de 10x10m na RPPNVE, foi contabilizado o número de forófitos analisados, para que nas investigações da diversidade de líquens nos demais pontos de coleta o número de forófitos analisados fosse o mesmo, evitando a obtenção de falsos padrões de comparação.

Os líquens localizados desde a base dos forófitos, até uma altura máxima de 2 metros, foram identificados, o que determinou o número 
de espécies de líquens para cada ponto de coleta. Todo material coletado foi devidamente acondicionado em embalagens identificadas com etiquetas constando a data e o local da coleta. As espécies facilmente reconhecidas em campo foram apenas identificadas e tiveram seu registro fotográfico, evitando-se coletas desnecessárias. As amostras colhidas foram utilizadas nos processos de identificação dos respectivos líquens e após a conclusão do presente estudo foram destinadas a compor o acervo técnico científico da Associação de Preservação e Pesquisa Ambiental Vale Encantado (APPAVE).

\section{PROCEDIMENTOS DE IDENTIFICAÇÃO DAS AMOSTRAS}

Para a identificação das amostras foram utilizadas chaves de sistemática de líquens obtidas no Grupo Latino-Americano de Liquenologia, bem como catálogos de imagens específicos para estudos e identificação de líquens. As amostras foram analisadas em microscópio estereoscópico (50x) e lupa estereoscópica (60x), tendo sido realizadas observações morfológicas detalhadas.

Durante o processo de identificação, foram observadas as características que possuem importância taxonômica descritas por Marcelli (2006).

Para gêneros em que se identificou apenas um indivíduo, não se prosseguiu a estudos químicos e histoquímicos mais detalhados, uma vez que a identificação da espécie não alteraria o resultado final do presente trabalho.

\section{análise dos dados}

Para a confecção das tabelas e interpretação dos dados foi utilizado software (freeware) Sistema de Análise de Vegetação Arbórea Nativa - SAVAN, versão 1.1, desenvolvido por profissionais da Universidade Federal do Rio Grande do Sul - UFRGS, disponibilizado gratuitamente pela referida instituição de ensino.

Para a análise da Frequência Absoluta foi adotada a seguinte fórmula: $\mathrm{FA}=100 * \mathrm{p} / \mathrm{P}$, onde $\mathrm{FA}=$ frequência absoluta da espécie; $\mathrm{p}=$ número de unidades amostrais em que ocorre a espécie e $\mathrm{P}=$ número total de unidades amostrais. Para o cálculo de Frequência Relativa foi adotada a seguinte fórmula: $\mathrm{FR}=100 * \mathrm{Fa} / \mathrm{Fa}$, onde $\mathrm{FR}=$ frequência relativa da espécie e $\mathrm{FA}=$ frequência absoluta da espécie. Para o cálculo do Índice de
Valor de Importância (IVI), foi adotada a seguinte fórmula: IVI = DR + DoR + FR, onde: $\mathrm{DR}=$ densidade relativa da espécie; $\mathrm{DoR}=$ dominância relativa da espécie e $\mathrm{FR}$ = frequência relativa da espécie.

Para a análise de similaridade entre os sete pontos de coleta foram utilizados o índice de Sörensen, que é dado pela fórmula: Is $=2 \mathrm{c} /(\mathrm{a}+$ b), onde: Is = índice de similaridade de Sörensen; $\mathrm{c}=$ número de espécies comuns a ambos os locais comparados; $\mathrm{a}=$ número de espécies ocorrentes no local $\mathrm{A} ; \mathrm{b}=$ número de espécies ocorrentes no local $\mathrm{B}$, bem como o índice de similaridade de Jaccard que é dado pela fórmula: $\mathrm{ISj}=\mathrm{c} /(\mathrm{a}+$ $b-c)$, onde: $c=$ numero de espécies comuns em ambas as amostras; $a=$ numero total de espécies em uma das amostras; $b=$ numero total de espécies na outra amostra.

\section{resultados e discussão}

Foram identificadas 42 espécies de líquens (TABELA 1), as quais se distribuíam em 23 gêneros e 14 famílias. A maioria das espécies (52,39\%) é representada pelos líquens foliosos, seguida dos líquens crostosos $(33,33 \%)$, líquens fruticosos $(9,52 \%)$ e liquens crostosos-fruticosos (4,76\%).

Todas as espécies identificadas estavam presentes na RPPN - Vale Encantado, e diante de tal fato, os dados deste ponto de coleta foram tomados como referência para fins de comparação com os demais pontos de coletas. Era esperado que no primeiro ponto de coletas (RPPN - Vale Encantado), a diversidade de líquens fosse de fato superior aos demais pontos de coletas em virtude da mesma se tratar de uma Unidade de Conservação distante do perímetro urbano e sem indústrias em sua proximidade, logo, pouco afetada pela poluição atmosférica.

Nos pontos de coletas situados no perímetro urbano foram observados resultados semelhantes, diferindo e merecendo especial atenção os pontos de coleta 4 (Praça Henrique Krugger) e 6 (Parque de Exposições Fernando Costa), nos quais a diversidade de líquens foi notadamente mais baixa que nos demais pontos de coleta dentro do perímetro urbano. Isso sugere que estas áreas recebam maior carga de poluentes atmosféricos do que as demais.

No último ponto de coleta, situado no Distrito Industrial III (P7), foi observada a menor diversidade liquênica, sendo identificadas apenas três espécies de líquens, sendo possível sugerir que a poluição atmosférica nesse ponto deve alcançar índices que tornam inviáveis a sobrevivência e 
Tabela 1: Diversidade de líquens observada em diferentes pontos de coletas na cidade de Uberaba - MG; P1 = RPPNVE; P2 = Entroncamento das Avenidas Teófilo Lamounier e Dr Thomaz Bawden de Camargos; P3 = Praça da Mogiana; P4 = Praça Henrique Krugger; P5 = Parque Municipal Mata do Ipê; P6 = Parque de Exposições Fernando Costa; P7 = Distrito Industrial III.

\begin{tabular}{llllllllll}
\hline TÁXON & \multicolumn{8}{c}{ PONTOS DE COLETA E REGISTROS } & HÁBITO \\
& P1 & P2 & P3 & P4 & P5 & P6 & P7 & \\
\hline
\end{tabular}

\section{ARTHONIACEAE}

Cryptothecia candida

Cryptothecia striata

$\begin{array}{lllll}x & x & x & x & \\ x & & & & x\end{array}$

Crostoso

BIATORACEAE

Bacidia fraxinea

Bacidia Scweinitzii

Bacidia sp

$x$

$x$

Crostoso

\section{CANDELARIACEAE}

Candelariella $s p$

Crostoso

$x$

$x$

Crostoso

Crostoso

\section{CHRYSOTRICACEAE}

Chrysothrix sp

$\begin{array}{llllll} & x & x & x & x\end{array}$

\section{CLADONIACEAE}

\section{Cladonia $s p^{1}$}

Cladonia $s p^{2}$

COCCOCARPIACEAE

Coccocarpia sp.

\section{COLLEMATACEAE}

Collema sp.

Leptogium $s p$

\section{GYALECTACEAE}

Coenogonium sp

\section{LECANORACEAE}

Lecanora $s p^{1}$

Lecanora $s p^{2}$

Lecanora $s p^{3}$

Pyrrhospora russula

\section{PARMELIACEAE}

Bulbothix isidiza

Canoparmelia sp.

Canoparmelia texana

Parmelia sp.

Parmelinopsis minarum

Parmotrema aurantiacoparum

Parmotrema chinense

Parmotrema conferendum $x$

$\mathrm{x}$

$\mathrm{X}$

X

$x$

$\mathrm{X}$

$x$

X

X $x \quad x$

$\mathrm{X}$

$x$

$\mathrm{X} \times$

$x \quad x$

$x \quad x$

$\begin{array}{lllllll}x & x & x & x & x & x & x\end{array}$

$x \quad x \quad x$

$\mathbf{x}$

$x$

$\mathrm{X}$

$x$
Crostoso

\section{Crostoso}

Crostoso-fruticoso

Crostoso-fruticoso

Folioso

Folioso

Crostoso

Crostoso

Crostoso

Crostoso

Crostoso

Crostoso

Folioso

Folioso

Folioso

Folioso

Folioso

Folioso

Folioso

Folioso 
Tabela 1: continuação...

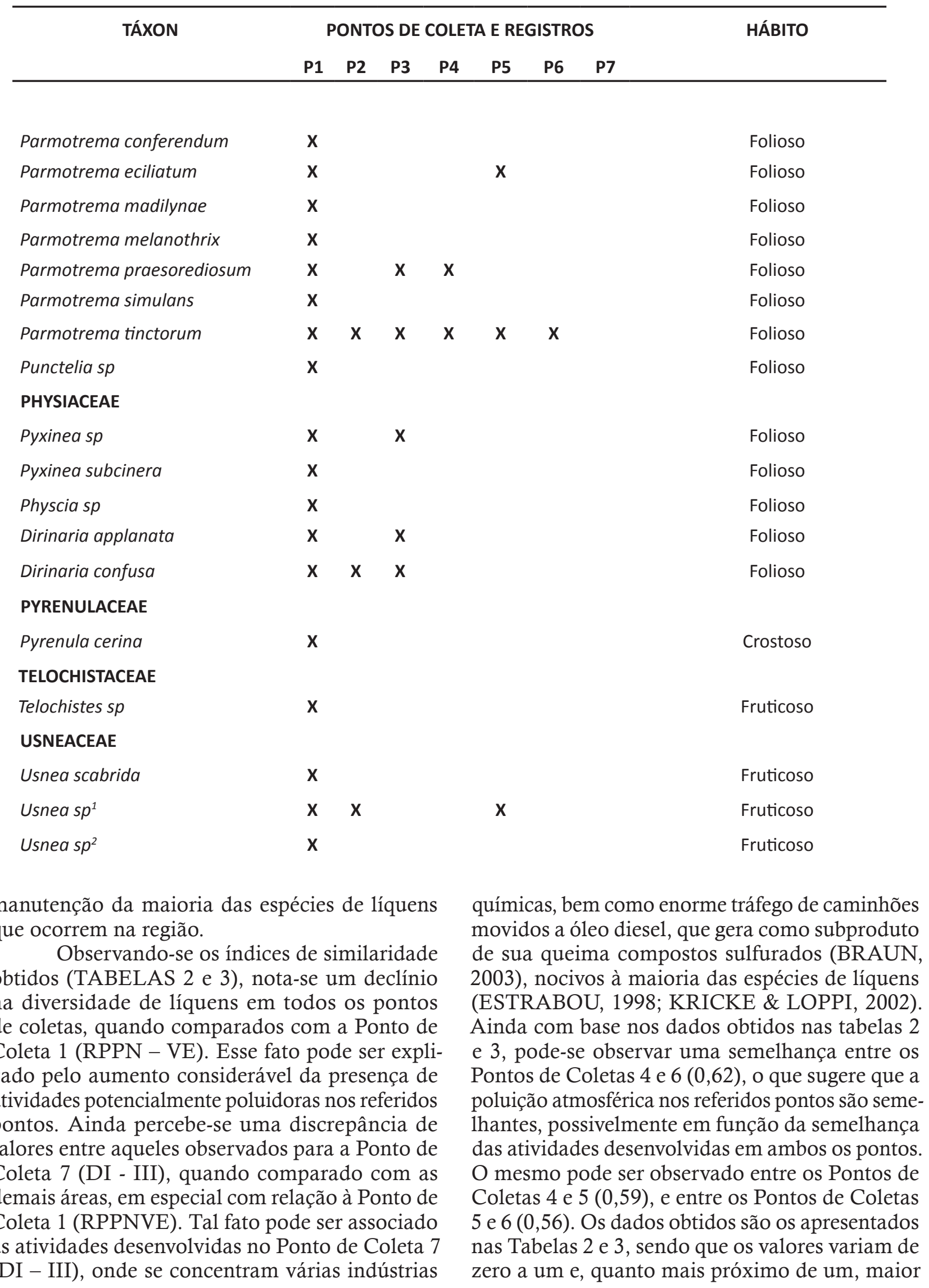


Tabela 2. - Índice de similaridade de Sorensen obtido entre a flora liquênica de diferentes pontos de coleta na cidade de Uberaba - MG. P1 = RPPNVE; P2 = Entroncamento das Avenidas Teófilo Lamounier e

Dr Thomaz Bawden de Camargos; P3 = Praça da Mogiana; P4 = Praça Henrique Krugger; P5 = Parque

Municipal Mata do Ipê; P6 = Parque de Exposições Fernando Costa; P7 = Distrito Industrial III

\begin{tabular}{cccccccc}
$\begin{array}{l}\text { Pontos } \\
\text { de Coleta }\end{array}$ & P1 & P2 & P3 & P4 & P5 & P6 & P7 \\
\hline P1 & 1,00 & 0,39 & 0,38 & 0,26 & 0,35 & 0,29 & 0,14 \\
P2 & 0,39 & 1,00 & 0,48 & 0,38 & 0,38 & 0,35 & 0,15 \\
P3 & 0,38 & 0,48 & 1,00 & 0,47 & 0,36 & 0,33 & 0,14 \\
P4 & 0,26 & 0,38 & 0,47 & 1,00 & 0,59 & 0,62 & 0,44 \\
P5 & 0,35 & 0,38 & 0,36 & 0,59 & 1,00 & 0,56 & 0,43 \\
P6 & 0,29 & 0,35 & 0,33 & 0,62 & 0,56 & 1,00 & 0,60 \\
P7 & 0,14 & 0,15 & 0,14 & 0,44 & 0,43 & 0,60 & 1,00 \\
\hline
\end{tabular}

a similaridade entre os pontos de coletas.

Com base na análise da distribuição das espécies, foi possível apontar quais espécies se mostraram mais presentes nos diversos pontos de coletas. Por exemplo, Canoparmelia texana esteve presente em todos os pontos, enquanto Parmotrema tinctorum e Chrysothrix sp estiveram presentes em seis dos sete pontos de coletas. Houve também aquelas cuja presença se limitou a alguns, ou apenas um ponto de coleta, tais como Bacidia Schweinitzii, Cladonia sp1, Coccocarpia sp, Collema sp, Lecanora sp2, Leptogium sp, Par- motrema aurantiacoparum, Parmotrema chinense, Parmotrema conferendum, Parmotrema madilynae, Parmotrema melanothrix, Parmotrema simulans, Physcia sp, Punctelia sp, Pyrenula cerina, Pyxinea subcinera, Telochistes sp, Usnea scabrida, todas presentes apenas no ponto de coleta P1. Analisando ainda a distribuição das famílias (Tabela 4), percebe-se com maior clareza que algumas famílias ocorreram somente no ponto de coleta 1 , tais como Collemataceae, Pyrenulaceae, Telochistaceae e Coccocarpiaceae.

Tabela 3. - Índice de similaridade de Jaccard obtido entre a flora liquênica de diferentes pontos de coleta na cidade de Uberaba - MG. P1 = RPPNVE; P2 = Entroncamento das Avenidas Teófilo Lamounier e Dr Thomaz Bawden de Camargos; P3 = Praça da Mogiana; P4 = Praça Henrique Krugger; P5 = Parque Municipal Mata do Ipê; P6 = Parque de Exposições Fernando Costa; P7 = Distrito Industrial III

\begin{tabular}{cccccccc}
$\begin{array}{c}\text { Pontos } \\
\text { de Coleta }\end{array}$ & P1 & P2 & P3 & P4 & P5 & P6 & P7 \\
\hline P1 & 1,00 & 0,24 & 0,24 & 0,15 & 0,21 & 0,17 & 0,07 \\
P2 & 0,24 & 1,00 & 0,31 & 0,23 & 0,24 & 0,21 & 0,08 \\
P3 & 0,24 & 0,31 & 1,00 & 0,31 & 0,22 & 0,20 & 0,08 \\
P4 & 0,15 & 0,23 & 0,31 & 1,00 & 0,42 & 0,44 & 0,29 \\
P5 & 0,21 & 0,24 & 0,22 & 0,42 & 1,00 & 0,38 & 0,27 \\
P6 & 0,17 & 0,21 & 0,20 & 0,44 & 0,38 & 1,00 & 0,43 \\
P7 & 0,07 & 0,08 & 0,08 & 0,29 & 0,27 & 0,43 & 1,00 \\
\hline
\end{tabular}


Tabela 4. - Análise da distribuição das Famílias de liquens identificadas em diferentes pontos de coletas na cidade de Uberaba - MG. 01 = RPPNVE; 02 = Entroncamento das Avenidas Teófilo Lamounier e Dr Thomaz Bawden de Camargos; 03 = Praça da Mogiana; 04 = Praça Henrique Krugger; 05 = Parque Municipal Mata do Ipê; 06 = Parque de Exposições Fernando Costa; 07 = Distrito Industrial III;

\begin{tabular}{lccc}
\multicolumn{1}{c}{ Família } & Indivíduos & \%Total & Unidades Amostrais \\
\hline PARMELIACEAE & 36 & 40,45 & $01,02,03,04,05,06,07$ \\
PHYSIACEAE & 9 & 10,11 & $01,02,03$ \\
LECANORACEAE & 9 & 10,11 & $01,02,03,04,05$ \\
BIATORACEAE & 8 & 8,99 & $01,04,05,06,07$ \\
CHRYSOTRICACEAE & 6 & 6,74 & $01,02,03,04,05,06$ \\
USNEACEAE & 5 & 5,62 & $01,02,05$ \\
ARTHONIACEAE & 4 & 4,49 & $01,03,05,06$ \\
CLADONIACEAE & 3 & 3,37 & 01,03 \\
GYALECTACEAE & 2 & 2,25 & 01,03 \\
COLLEMATACEAE & 2 & 2,25 & 01 \\
CANDELARIACEAE & 2 & 2,25 & 01,02 \\
PYRENULACEAE & 1 & 1,12 & 01 \\
TELOCHISTACEAE & 1 & 1,12 & 01 \\
COCCOCARPIACEAE & 1 & 1,12 & 01 \\
\hline
\end{tabular}

Com base na distribuição das espécies de líquens pelos diferentes pontos de coletas, foi possível analisar diferentes índices sociológicos de interesse, sendo que para o presente trabalho foi dado ênfase às frequências e valores de impor- tância, uma vez que os mesmos puderam apontar com eficiência a predominância da presença de determinadas espécies, como pode ser visto na Tabela 5.

Tabela 5. - Análise liquenossociológica das espécies identificadas nos diferentes pontos de coletas na cidade de Uberaba - MG. FA: Frequência Absoluta; FR: Frequência Relativa; IVI: Índice de Valor de Importância.

\section{Espécies}

Bacidia Scweinitzii

Bacidia fraxinea

Bacidia sp

Bulbothix isidiza

Candelariella sp

Canoparmelia sp.

Canoparmelia texana

Chrysothrix sp

Cladonia sp1

Cladonia sp2

Coccocarpia sp.

Coenogonium sp
FA

14,29

28,57

71,43

28,57

28,57

28,57

100,00

85,71

14,29

28,57

14,29

28,57
FR

1,12

2,25

5,62

2,25

2,25

2,25

7,87

6,74

1,12

2,25

1,12

2,25
IVI

2,25

4,49

11,24

4,49

4,49

4,49

15,73

13,48

2,25

4,49

2,25

4,49 
Tabela 5. - continuação...

\begin{tabular}{|c|c|c|c|}
\hline Espécies & FA & FR & IVI \\
\hline Collema sp. & 14,29 & 1,12 & 2,25 \\
\hline Cryptothecia candida & 28,57 & 2,25 & 4,49 \\
\hline Cryptothecia striata & 28,57 & 2,25 & 4,49 \\
\hline Dirinaria applanata & 28,57 & 2,25 & 4,49 \\
\hline Dirinaria confusa & 42,86 & 3,37 & 6,74 \\
\hline Flavoparmelia sp. & 28,57 & 2,25 & 4,49 \\
\hline Lecanora sp1 & 42,86 & 3,37 & 6,74 \\
\hline Lecanora sp2 & 14,29 & 1,12 & 2,25 \\
\hline Lecanora sp3 & 28,57 & 2,25 & 4,49 \\
\hline Leptogium $s p$ & 14,29 & 1,12 & 2,25 \\
\hline Parmelinopsis minarum & 57,14 & 4,49 & 8,99 \\
\hline Parmotrema aurantiacoparum & 14,29 & 1,12 & 2,25 \\
\hline Parmotrema chinense & 14,29 & 1,12 & 2,25 \\
\hline Parmotrema conferendum & 14,29 & 1,12 & 2,25 \\
\hline Parmotrema eciliatum & 28,57 & 2,25 & 4,49 \\
\hline Parmotrema madilynae & 14,29 & 1,12 & 2,25 \\
\hline Parmotrema melanothrix & 14,29 & 1,12 & 2,25 \\
\hline Parmotrema reticulatum & 42,86 & 3,37 & 6,74 \\
\hline Parmotrema simulans & 14,29 & 1,12 & 2,25 \\
\hline Parmotrema tinctorum & 85,71 & 6,74 & 13,48 \\
\hline Physcia sp & 14,29 & 1,12 & 2,25 \\
\hline Punctelia sp & 14,29 & 1,12 & 2,25 \\
\hline Pyrenula cerina & 14,29 & 1,12 & 2,25 \\
\hline Pyrrhospora russula & 42,86 & 3,37 & 6,74 \\
\hline Pyxinea sp & 28,57 & 2,25 & 4,49 \\
\hline Pyxinea subcinera & 14,29 & 1,12 & 2,25 \\
\hline Telochistes sp & 14,29 & 1,12 & 2,25 \\
\hline Usnea scabrida & 14,29 & 1,12 & 2,25 \\
\hline Usnea sp1 & 42,86 & 3,37 & 6,74 \\
\hline Usnea sp2 & 14,29 & 1,12 & 2,25 \\
\hline
\end{tabular}

Há de se destacar, de acordo com a Tabela 5 , a presença em todos os pontos de coletas $(\mathrm{FA}=$ 100,00), inclusive na unidade amostral denominada P7, da espécie Canoparmelia texana (PARMELIACEAE), cujo Índice de Valor de Importância foi o maior dentre as espécies identificadas (IVI= 15,73), descrita por Coccaro et al. (2000) e Saiki et al. (2003), como tolerante à poluição atmosférica. (Ver Tabela 1). Esta espécie, segundo Marcelli (1998), vem sendo utilizada em São Paulo em estudos relacionados à poluição atmosférica $e$, ainda segundo Martins et al. (2008) é apontada como uma espécie favorecida em ambientes alterados.

\section{CONCLUSÃO}

Com base na análise dos dados levantados no presente estudo, tendo como foco o estudo da diversidade liquênica, é possível concluir que entre referidas áreas aquela que apresentou maior diversidade de líquens e que possivelmente pos- 
sui o menor nível de poluentes atmosféricos é a denominada P1 (RPPNVE). Já a unidade amostral que apresentou a menor diversidade liquênica e provavelmente possui o maior nível de poluição do ar foi aquela denominada P7 (DI - III).

Ainda, é possível concluir que quando comparados com o primeiro ponto de coleta (P1 RPPNVE), todos os demais pontos devem possuir qualidade do ar inferior ao mesmo, uma vez que observou-se em todos eles um declínio acentuado da diversidade de líquens, em especial nos pontos P4 (Praça Henrique Krugger), e P6 (Parque de Exposições Fernando Costa - ABCZ).

Desta forma, diante do baixo custo do monitoramento passivo da qualidade do ar utilizando-se líquens como bioindicadores, este trabalho, além de ser pioneiro para a cidade de Uberaba - MG, pode contribuir para o delineamento das políticas públicas voltadas para o zoneamento do município, bem como para o controle de poluição atmosférica, levando-se em consideração o conhecimento acerca das áreas onde esse tipo de poluição se mostra mais evidente.

\section{REFERÊNCIAS}

API, 2011, em EDITORA ABRIL; Planeta Sustentável, , disponível em <http://planetasustentavel. abril.com.br/noticia/desenvolvimento/fosseis-companhias-petroliferas-futuro-mais-eletrico-quatrorodas-545227.shtml> ; Data de acesso:17 de maio 2011;

APTROOT, A.; SPARRIUS, L.; Pictures of tropical lichens; Disponível em <www.tropicallichens.net> ; Data de acesso: 12 de fev. de 2012;

BISSACOT, S.; MACHADO, S. R.; MARCELLI, M. P.; Thallus anatomy of Canoparmelia texana (Parmeliaceae, lichenized Ascomycota); Biota Neotrop. Vol. 10, n 3, Campinas July/Sept. 2010;

BRAUN, S>; APPEL, L. G.; SCHMAL, M.; 2003; A poluição gerada por máquinas de combustão interna movidas à diesel - a questão dos particulados. Estratégias atuais para a redução e controle das emissões e tendências futuras; Quim. Nova, Vol. 27, No. 3, 472-482, 2003;

BRUNIALTI, G. e GIORDANI, P. 2003. Variability of lichen diversity in a climatically heterogeneous area (Liguria, NW Italy). Lichenologist 35: 55-69;
CARNEIRO, R. M A. Bioindicadores vegetais na poluição atmosférica: uma contribuição para a saúde da comunidade. 2004. 146p. Dissertação (Mestrado) - Escola de Enfermagem de Ribeirão Preto, universidade de São Paulo, Ribeirão Preto;

COCCARO, D.M.B, SAIKI, M.B.A., VASCONCELOS, M.P. \& MARCELLI, M.P. 2000. Analysis of Canoparmelia texana lichens collected in Brazil by neutron activation analysis. In: Biomonitoring of atmospheric pollution (with emphasis on trace elements), BioMAP. International Atomic Energy Agency, Lisboa, pp. 143-148;

ELIASARO, S. et al.; Levantamento da flora liquênica da Ilha do Mel, Paraná, Departamento de Botânica da Universidade Federal do Paraná, 2010;

ELIASARO S., VEIGA P. W., DONHA C. G., NOGUEIRA L. 2009, Inventário de macrolíquens epí $\square$ tos sobre árvores utilizadas na arborização urbana em Curitiba, Paraná, Brasil: Subsídio para biomonitoramento urbano, Biotemas, 22 (4): 1-8, dezembro de 2009;

ESTRABOU, C. 1998. Lichen species identification and distribution according tolerance to airbone contamination in the city of Córdoba (Argentina). In: M.P. Marcelli \& M.R.D. Seaward (eds.). Lichenology in Latin America: history, current knowledge and application. CETESB, São Paulo, pp. 165-169;

GIORDANI, P. 2007. Is the diversity of epiphytic lichens a reliable indicator of air pollution? A case study from Italy. University of Genova, Genova, Italy; Environmental Pollution, 146 (2): 317-323;

GUNNARSSON, B., HAKE, M. e HULTENGREN, S. 2004. A functional relationship between species richness of spiders and lichens in spruce. Biodiversity and Conservation 13:685-693;

IBGE - Instituto Brasileiro de Geografia e Estatística, disponível em <http://www.ibge.gov.br/cidadesat/painel/painel.php?codmun=317010\#>, Data de acesso: 12 de mar. de 2012;

KÖPPEN, W. 1948. Climatologia Ed. Fondo Cultural Economica, Mexico City;

KRICKE, R. \& LOPPI, S. 2002. Bioindication: The I.A.P. approach. In: P.L Nimis, C. Scheidegger $\&$ P.A. Wolseley (eds.). Monitoring with lichensMonitoring lichens. Kluwer Academic Publishers. 
Dordrecht, pp. 21-38;

LÜCKING, R. 1995. Biodiversity and conservation of foliicolous lichens in Costa Rica. Mitteilungen der Eidgenössischen Forschungsanstalt für Wald, Schnee und Landschaft 70:63-92.

MARANTE, F. J. T.; CASTELLANO, A. G.; OYOLA, F. L.; BARRERA, J. B.; Ecología Química En Hongos Y Líquenes, Rev. Acad. Colomb. Cienc, volumen XXVI I I, número 109-diciembre de 2004;

MARCELLI, M. P. 1998. Hystory and current knowledge of Brazilian lichenology. In: Marcelli, M. P. \& Seaward, M. R. D. (Eds.). Lichenology in Latin America: History, current knowledge and application. CETESB, São Paulo, Brasil, p.25-45;

MARCELLI, M. P. 2002. Checklist of lichens and lichenicolous fungi of Brazil. Version 1, disponível em <http://www.biologie.uni-hamburg.de/checklist/ brazil_1.htm> ; Data de acesso 03 de maio 2011;

MARCELLI, M. P., 2006. Cáp. I Fungos Liquenizados. Em XAVIER FILHO, L. et al. Biologia de Líquens. Rio de janeiro, Brasil, p. 24-74;

MARTINS, S. M. A.; KÄFFER, M. I.; LEMOS, A. 2008; Liquens como bioindicadores da qualidade do ar numa área de termoelétrica, Rio Grande do Sul, Brasil. Hoehnea 35 (3): 425-433;

NASH III, T. H. 1996. Photosynthesis, respiration, productivity and growth. In: Nash III, T. H. (ed), Lichen biology. Cambridge University Press, Cambridge, pp 88-135;

PREFEITURA MUNICIPAL DE UBERABA, Portal eletrônico, disponível em: <http://www.uberaba. mg.gov.br/portal/conteudo,1030>, Data de acesso em 17 de maio 2011;

RAIMUNDO COSTA, W.; FARIA, J.E. Estudos preliminares para eventual caracterização de encrave Mata Atlântica-Cerrado nas áreas da RPPN-Vale Encantado, Uberaba, MG.. Em: Anais do $1^{\circ}$ Congresso de Meio Ambiente Vale Encantado Cerrado - Diversidade e Recursos, 2011. Uberaba, MG. P. 57-66.

RAVEN, Peter H; EICHHORN, Susan E.; EVERT, Ray F. Biologia Vegetal, cap. 11. $6^{\mathrm{a}}$. Ed. Rio de Janeiro: Guanabara Koogan, 2001;
SAIKI, M., FUGA, A., ALVES, E.R., VASCONCELLOS, M.B.A. \& MARCELLI, M. 2003; The use of Canoparmelia texana lichenized fungi in the study of atmospheric air pollution, In: Third International Workshop on Biomonitoring of Atmospheric Pollution, Ljubljana, pp. 705-708;

SILLET, S. C., MAcCUNE, B., PECK, J.E., RAMBO, T. R. e RUCHTY, A. 2000. Dispersal limitations of epiphytic lichens result in species dependent on old-growth forests. Ecological Applications 10: $789-799$.

SILVA, J. W.; GUIMARÃES, E. C.;TAVARES, M.; 2003. Variabilidade temporal da precipitação mensal e anual na estação climatológica de Uberaba-MG, Ciênc. Agrotec. vol.27 nº.3 Lavras May/June 2003;

SOMMERFELDT, M.: JOHN, V. 2000. Evaluation of a method for the reassessment of air quality by lichen mapping in the city of Izmir, Turkey. Turkey Journal of Botany, 25 (2001): 45-5

SPIELMANN, A. A., et al. Parmeliaceae (Ascomicota liquenizados) nos barrancos e peraus da Encosta da Serra Geral, Vale do Rio Pardo, Rio Grande do Sul, Brasil. IHERINGIA, Sér. Bot., Porto Alegre, v 63, n 1, p. 159 - 169, jan - jun, 2008.

VALENCIA, M. C de, CEBALLOS, J. A., 2002. Hongos Líquenizados. Universidad Nacional de Colômbia, Bogotá; 2002; 